\title{
Herbal Sunscreens and Photo Protection Compositions: A
}

\section{Patent Review}

\section{Singh $\mathrm{S}^{*}$ and Sharma N \\ Chitkara College of Pharmacy, Chitkara University, India}

*Corresponding author: Sukhbir Singh, Chitkara College of Pharmacy, Chitkara University, Chandigarh Patiala National Highway (NH-64), Patiala-140401, Punjab, India, Email: singh.sukhbir12@gmail.com

\section{Review Article \\ Volume 1 Issue 8}

Received Date: November 23, 2017

Published Date: December 02, 2017

\begin{abstract}
Solar ultraviolet type B radiations (UVB) is fundamentally liable to be absorbed by keratinocytes leading to production of free radicals or reactive oxygen species (ROS) which might cause skin erythema, sunburn, skin cancer and premature ageing. Herbal sunscreen guard skin from sun's radiations which reduces sunburns with reduced risk of skin harms. Antioxidants quench free radicals and ROS which might aid photo-protection effect. This review compiled previously patented herbal photo protection compositions and sunscreens.
\end{abstract}

Keywords: Skin Erythema; Sunscreen; Photo Protection

Abbreviations: UVB: Ultraviolet Type B; ROS: Reactive Oxygen Species

\section{Introduction}

Solar ultraviolet radiations at the earth's surface encompass approximately 95-98 \% UVA and 2-5 \% UVB. The frequency of ultraviolet type $B$ is fundamentally liable to be absorbed by keratinocytes leading to production of free radicals or reactive oxygen species (ROS) which might cause skin erythema, sunburn, skin cancer and premature ageing [1,2]. Anti-oxidants quench free radicals and ROS which might aid photo-protection effect. Herbal sunscreen topical products guard skin from sun's radiations which reduces sunburns with reduced risk of skin harms. This review compiled previously patented herbal photo protection compositions and sunscreens. Few herbal photo protectants have been represented in Figure 1.

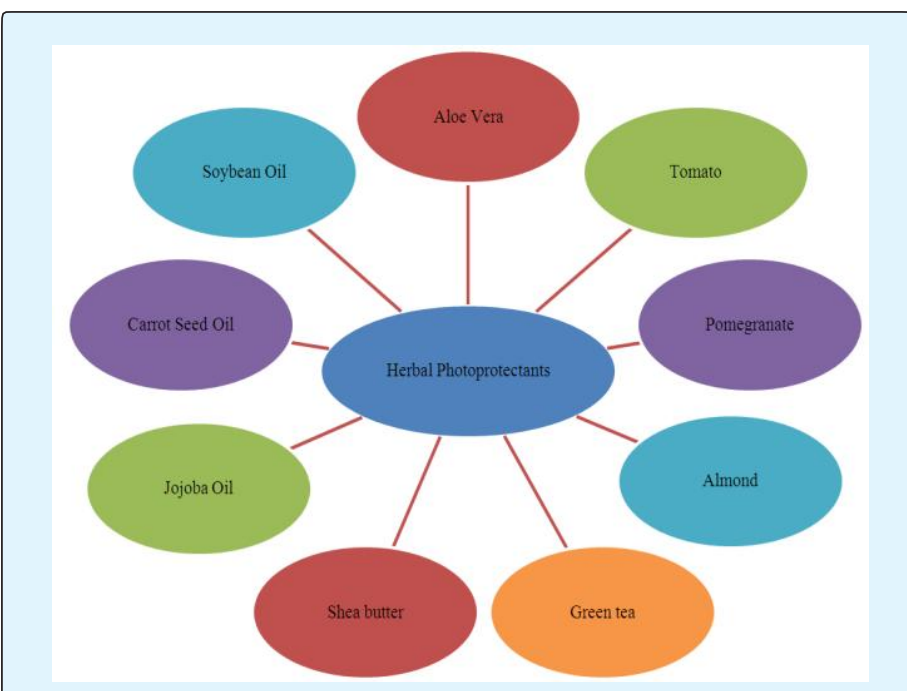

Figure 1: Examples of herbal photo protectants. 


\section{Open Access Journal of Pharmaceutical Research}

\section{Ultimate Characteristics of Sunscreen} Product

A high-quality sunscreen ought to absorb or riddle out the sunburn emission causing and must be non-toxic and non-irritating. It should be stable under air, light and moisture. Moreover, it must be moderately non-volatile, subsequently will not evaporate [3]. Herbal sun protection compositions and sunscreens patented for patented for sunscreen and photo protection activity has been listed in Table 1, 2, respectively.

\begin{tabular}{|c|c|c|c|}
\hline $\begin{array}{c}\text { Publication No./ Publication } \\
\text { date/ Applicant }\end{array}$ & Title of patent & Disclosure & Reference \\
\hline $\begin{array}{l}\text { US } 7311896 \text { B2/ Dec 25, 2007/ } \\
\text { Mmi Corporation }\end{array}$ & $\begin{array}{c}\text { Natural sunscreen } \\
\text { fompositions and processes for } \\
\text { producing the same }\end{array}$ & $\begin{array}{l}\text { Extracts of Hedychium spicatum and/or } \\
\text { Alpinia galanga as natural sunscreen }\end{array}$ & [4] \\
\hline \multirow{3}{*}{$\begin{array}{l}\text { US } 20130243709 \text { A1/ } 19 \text { Sep } \\
\text { 2013/ James E. Hanson, Cosimo } \\
\text { Antonacci }\end{array}$} & \multirow{3}{*}{$\begin{array}{l}\text { Natural Sunscreen } \\
\text { Composition }\end{array}$} & $\begin{array}{l}\text { UV-blocking component comprising of: } \\
\text { - Natural extracts of milk thistle, white } \\
\text { willow, St John's wort, griffonia seed, } \\
\text { Galla chinensis, olive leaf, hops, gentian, } \\
\text { bilberry, chrysanthemum, coptis root, } \\
\text { magnolia bark, rhubarb, red clover, rose } \\
\text { hip, schisandra berry, valerian root and } \\
\text { grape seed. }\end{array}$ & \multirow{3}{*}[5]{} \\
\hline & & $\begin{array}{l}\text { - Natural oils of cinnamon bark, cocoa, } \\
\text { coffee, cognac, ravensara, tansy blue, } \\
\text { vanilla and yarrow. }\end{array}$ & \\
\hline & & $\begin{array}{l}\text { - Nutrients i.e. vitamin D, folic acid, } \\
\text { riboflavin, pyridoxine, cyanocobalamine, } \\
\text { collagen or partially hydrolyzed collagen, } \\
\text { silk protein or partially hydrolyzed silk } \\
\text { protein, thymine, cytosine, adenine, } \\
\text { guanine, rutin, quercetin, azalein, } \\
\text { hyperoside, isoquercetin, kampferitin, } \\
\text { myricitrin, robinin, speraeoside, } \\
\text { xanthorhamin, icariin, truxrutin, vitamin } \\
\text { K and coenzyme Q. }\end{array}$ & \\
\hline $\begin{array}{l}\text { US } 20050042186 \text { A1/ } 24 \text { Feb } \\
\text { 2005/ Peter Zahner }\end{array}$ & All natural sunscreen lotion & $\begin{array}{l}\text { Natural topical sunscreen composition } \\
\text { includes } 0.01 \% \text { to } 5 \% \text { by weight of } \\
\text { biological melanin, } 2 \% \text { to } 10 \% \text { by weight } \\
\text { of green tea extract with polyphenol } \\
\text { content greater than } 40 \% \text { and } 2 \% \text { to } 10 \% \\
\text { by weight of either titanium dioxide } \\
\text { and/or zinc oxide. }\end{array}$ & {$[6]$} \\
\hline $\begin{array}{l}\text { US } 5817299 \text { A/ } 6 \text { Oct 1998/ E-L } \\
\text { Management Corp. }\end{array}$ & $\begin{array}{l}\text { Non-chemical sunscreen } \\
\text { composition }\end{array}$ & $\begin{array}{c}\text { Non-chemical sunscreen comprising } \\
\text { about } 50 \% \text { by weight of } \\
\text { proanthocyanidins, } \gamma \text {-oryzanol, ferulic } \\
\text { acid, titanium dioxide and scutellaria } \\
\text { extract. }\end{array}$ & [7] \\
\hline $\begin{array}{l}\text { WO 2012055678 A1/ } 3 \text { May } \\
\text { 2012/ Unilever Nv, Unilever Plc, } \\
\text { Hindustan Unilever Limited }\end{array}$ & $\begin{array}{l}\text { A stable sunscreen } \\
\text { composition }\end{array}$ & $\begin{array}{l}\text { Discloses a stable sunscreen containing } \\
\text { zingerone as sunscreen stabilizer which } \\
\text { is a derivative of gingerol found in ginger. }\end{array}$ & [8] \\
\hline
\end{tabular}

Table 1: Herbal photo protection compositions patented for sunscreen activity. 


\begin{tabular}{|c|c|c|c|}
\hline $\begin{array}{c}\text { Publication No./ Publication } \\
\text { date/ Applicant }\end{array}$ & Title of patent & Disclosure & Reference \\
\hline $\begin{array}{l}\text { EP } 0953337 \text { A1/ } 3 \text { Nov 1999/ } \\
\text { Ioannis Papadakos }\end{array}$ & Preparation of sun protection oil & $\begin{array}{l}\text { Sun protection oil is prepared with } \\
\text { pure virgin olive oil in combination } \\
\text { with vitamins E and A. }\end{array}$ & [9] \\
\hline $\begin{array}{l}\text { US 20050112156 A1/ } 26 \text { May } \\
\text { 2005/ Stefan Busch, Rolf Kawa, } \\
\text { Alfred Westfechtel }\end{array}$ & Sun protection compositions & $\begin{array}{l}\text { New sun protection compositions } \\
\text { with improved water resistance } \\
\text { have an effective content of diol } \\
\text { dimer fatty acid esters and dimer } \\
\text { diol dimer fatty acid esters. }\end{array}$ & [10] \\
\hline $\begin{array}{c}\text { WO 2014016349 A1/ Jan 30, } \\
\text { 2014/ Biosynthis }\end{array}$ & Photo protective composition & $\begin{array}{l}\text { Photo-protective containing karanja } \\
\text { oil and ioned polyester. }\end{array}$ & {$[11]$} \\
\hline $\begin{array}{c}\text { US 20080138446 A1/ } 12 \text { June } \\
\text { 2008/ Lavender Hill Projects Pvt } \\
\text { Ltd }\end{array}$ & Herbal composition & $\begin{array}{c}\text { Herbal composition containing } \\
\text { extracts from Thuja, Poke, Sweet } \\
\text { violet, Red clover, Greater celandine } \\
\text { and Calendula. }\end{array}$ & {$[12]$} \\
\hline $\begin{array}{c}\text { US } 7344728 \text { B1/ Mar 18, 2008/ } \\
\text { Perry Stephen C }\end{array}$ & $\begin{array}{l}\text { Insect repellent with sun } \\
\text { protection factor }\end{array}$ & $\begin{array}{l}\text { An insect repellent composition } \\
\text { which includes natural oils } \\
\text { (citronella and orange), synthetic } \\
\text { ingredients (D-limonene and phtalic } \\
\text { acid), sunscreen vitamin F and } \\
\text { volatile silicones. }\end{array}$ & [13] \\
\hline $\begin{array}{l}\text { EP 2144608 A1/ Jan 20, 2010/ } \\
\text { Sytheon Ltd. }\end{array}$ & $\begin{array}{l}\text { Sunscreen compositions and } \\
\text { methods }\end{array}$ & $\begin{array}{l}\text { Sunscreen compositions comprising } \\
\text { of UV-B or UV-A/UV-B sunblock } \\
\text { active, meroterpene (Bakuchiol) and } \\
\text { dermatological acceptable carrier. }\end{array}$ & {$[14]$} \\
\hline $\begin{array}{c}\text { US } 9173823 \text { B2/ } 3 \text { Nov 2015/ } \\
\text { Institut Biophytis Sas, Universite } \\
\text { Pierre Et Marie Curie }\end{array}$ & Preparation for sun protection & $\begin{array}{c}\text { Food preparation containing } \\
\text { norbixin, or bixin intended to be } \\
\text { administered orally for protection of } \\
\text { mammalian skin against UV- } \\
\text { radiation. }\end{array}$ & {$[15]$} \\
\hline \begin{tabular}{|c|} 
WO 2003020236 A2/13 Mar \\
2003/ Hadasit Medical Research \\
Services \& Development Limited, \\
Yissum Research Development \\
Company of The Hebrew \\
University of Jerusalem \\
\end{tabular} & $\begin{array}{l}\text { The utilization of natural } \\
\text { pigments from lichens, } \\
\text { cyanobacteria, fungi and plants } \\
\text { for sun protection }\end{array}$ & $\begin{array}{l}\text { Natural extract from fungus, } \\
\text { cyanobactria, plants, lichens or a } \\
\text { mixture having an ultra violet } \\
\text { absorbency in range of } 220 \mathrm{~nm} \text { to } \\
425 \mathrm{~nm} .\end{array}$ & {$[16]$} \\
\hline $\begin{array}{c}\text { US } 5614197 \text { A/ } 25 \text { Mar 1997/ } \\
\text { ndustrial Farmaceutica Cantabria } \\
\text { S.A. }\end{array}$ & $\begin{array}{l}\text { Polypodium extract as } \\
\text { photoprotectant }\end{array}$ & $\begin{array}{l}\text { Sunscreen containing extracts from } \\
\text { ferns of Polypodium. }\end{array}$ & {$[17]$} \\
\hline $\begin{array}{l}\text { US } 20090324522 \text { A1/ } 31 \text { Dec } \\
\text { 2009/ Western Holdings, Llc }\end{array}$ & Skin protectant compositions & $\begin{array}{l}\text { A topical sunscreen synergistic } \\
\text { composition includes extracts of } \\
\text { hibiscus flower, ferula assa foetida } \\
\text { root, pear fruit, and green tea leaf. }\end{array}$ & [18] \\
\hline $\begin{array}{c}\text { US } 4806344 \text { A/ } 21 \text { Feb 1989/ } \\
\text { Gaskin Frances C }\end{array}$ & $\begin{array}{l}\text { Sun protectant composition and } \\
\text { method }\end{array}$ & $\begin{array}{l}\text { The topical sunscreen composition } \\
\text { containing melanin as an active } \\
\text { ingredient. It also contains } \\
\text { sunscreens, vitamins and emollients. } \\
\text { These ingredients are rapidly } \\
\text { blended through ultrasound for } 2-3 \\
\text { hours to enhance sun protective } \\
\text { factor. }\end{array}$ & [19] \\
\hline
\end{tabular}




\section{Open Access Journal of Pharmaceutical Research}

\begin{tabular}{|c|c|c|c|}
\hline $\begin{array}{l}\text { US } 6235271 \text { B1/ } 22 \text { May 2001/ } \\
\text { Ciba Specialty Chemicals } \\
\text { Corporation }\end{array}$ & UV-protection formulation & $\begin{array}{l}\text { Sun protection agent comprising of } \\
\text { micronized organic UV absorber, an } \\
\text { oil-soluble, non-micronized UV } \\
\text { absorber and/or an inorganic micro } \\
\text { pigment; and polymeric hollow } \\
\text { sphere additive and/or xanthan } \\
\text { and/or polyvinylpyrrolidone. }\end{array}$ & [20] \\
\hline
\end{tabular}

Table 2: Herbal sunscreens patented for photo protection.

\section{Conclusion}

This patent review involves previously patented herbal photo protection compositions and sunscreens which are valuable to avert skin damage caused by acute or persistent exposure to solar UV radiations. This patent review was carried using the keywords herbal sunscreen, photo protection and photo ageing.

\section{References}

1. Mishra AK, Mishra A, Chattopadhyay P (2011) Herbal cosmeceuticals for photo protection from ultraviolet B radiation: A Review. Trop J Pharm Res 10(3): 351360.

2. Yusuf N, Irby C, Katiyar SK, Elmets CA (2007) Photo protective effects of green tea polyphenols. Photodermatol Photoimmunol Photomed 23(1): 4856.

3. Korać RR, Khambholja KM (2011) Potential of herbs in skin protection from ultraviolet radiation. Pharmacogn Rev 5(10): 164-173.

4. Mitra SK, Babu UV, Ranganna MV (2007) Natural sunscreen compositions and processes for producing the same. U.S. Patent 7311896 B2.

5. Hanson JE, Antonacci C (2013) Natural Sunscreen Composition. U.S. Patent 20130243709 A1.

6. Zahner P (2005) All natural sunscreen lotion. U.S. Patent 20050042186 A1.

7. Manirazman AM (1998) Non-chemical sunscreen composition. U.S. Patent 5817299 A.

8. Chavan MV, Kunjupillai B, Vaidya AA (2012) A stable sunscreen composition. World Intellectual Property Organization 2012055678 A1.
9. Papadakos I (1999) Preparation of sun protection oil. European Union patent 0953337 A1.

10. Busch S, Kawa R, Westfechtel A (2005) Sun protection compositions. U.S. Patent 20050112156 A1.

11. Bernoud T, Ramiandrasoa P (2014) Photoprotective composition. World Intellectual Property Organization 2014016349 A1.

12. Hilterman KA (2008) Herbal Composition. U.S. Patent 20080138446 A1.

13. Perry SC (2008) Insect repellent with sun protection factor. U.S. Patent 7344728 B1.

14. Chaudhuri RK (2010) Sunscreen compositions and methods. European Union patent 2144608 A1.

15. Veillet S, Lafont R, Dioh W (2015) Preparation for sun protection. U.S. Patent 9173823 B2.

16. Enk DC, Srebnik M, Lev O, Hochberg M, Dor I, et al. (2003) The utilization of natural pigments from lichens, cyanobacteria, fungi and plants for sun protection. World Intellectual Property Organization 2003020236 A2.

17. Pathak MA, Gonzalez S, Fitzpatrick TB (1997) Polypodium extract as photoprotectant. U.S. Patent 5614197 A.

18. Chevreau N (2009) Skin protectant compositions. U.S. Patent 20090324522 A1.

19. Gaskin FC (1989) Sun protectant composition and method. U.S. Patent 4806344 A.

20. Luther H, Stehlin A, Herzog B (2001) UV-protection formulation. U.S. Patent 6235271 B1. 